\section{THU0031 THERAPEUTIC TREATMENT OF ANTI-FRACTALKINE MONOCLONAL ANTIBODY INHIBITS JOINT DESTRUCTION IN COLLAGEN-INDUCED ARTHRITIS MODEL}

K. Hoshino-Negishi ${ }^{1}$, M. Ohkuro ${ }^{2}$, T. Nakatani ${ }^{1}$, W. Ikeda ${ }^{1}$, Y. Kuboi ${ }^{1}$, N. Ishii ${ }^{1}$ T. Yamauchi ${ }^{1}$, N. Yasuda ${ }^{1}$, T. Imai ${ }^{1} .{ }^{1}$ Research \& Development, KAN Research Institute. Inc., Kobe-Shi; ${ }^{2}$ Research Project Promotion Group, EA Pharma Co., Ltd., Kawasaki-ku, Japan

Background: In the Phase 1/2 clinical study, E6011, a novel humanized anti-fractalkine ( $F K N)$ monoclonal antibody (mAb) demonstrated a promising efficacy in active rheumatoid arthritis (RA) patients who were inadequately controlled by MTX and/or TNF-a inhibitors. However, the effect of anti-FKN $\mathrm{mAb}$ on joint destruction remains to be elucidated. In RA, synovium-infiltrated monocytes/macrophages cause synovitis and cartilage damage. Osteoclasts are generated from osteoclast precursor cells (OCPs) and cause bone erosion. FKN is expressed on endothelial cells and fibroblast-like synoviocytes in synovium in both experimental arthritis model and RA patients. FKN is also expressed on osteoblasts in neonatal mouse calvariae. CX3CR1, the receptor for FKN, is expressed on monocytes/macrophages and OCPs. Therefore, the interaction of FKN and CX3CR1 might play important roles in migration, differentiation and activation of these cells, leading to cartilage damage and bone erosion.

Objectives: We examined the efficacy of an anti-FKN mAb on collagen-induced arthritis (CIA) in mice, especially on joint destruction.

Methods: For the induction of $\mathrm{CIA}, \mathrm{DBA} / 1 \mathrm{~J}$ mice were immunized with bovine type II collagen. Anti-FKN mAb or control IgG was injected twice a week from the day of the 1st immunization (for the prophylactic treatment) or after the onset of CIA (for the therapeutic treatment). The clinical arthritis score was defined as the sum of the scores of four paws. Plasma concentrations of IL-6, TNF-a, serum amyloid A (SAA), Tartrate-Resistant Acid Phosphatase type $5 b$ (TRAP-5b), Cartilage Oligomeric Matrix Protein (COMP) and Matrix Metalloproteinase 3 (MMP-3) were measured using ELISA. Radiological score was measured by the soft x-ray images of limb bones. Alcian blue/Alizarin red and Tartrate-Resistant Acid Phosphatase (TRAP) staining were performed for histopathological analysis of ankle joints.

Results: In the prophylactic treatment, anti-FKN mAb markedly reduced the clinical arthritis score, soft $x$-ray score, and plasma levels of TRAP-5b, COMP and MMP-3, whereas plasma levels of IL-6, TNF-a and SAA were not significantly reduced compared with control IgG-treated mice. Histopathological analysis demonstrated almost complete suppression of joint destruction and dramatic reduction in the number of TRAP-positive cells by the treatment of anti-FKN mAb. Importantly, therapeutic treatment of anti-FKN mAb also significantly ameliorated clinical arthritis score and soft x-ray score. Synovitis, pannus formation, cartilage degradation and bone erosion were also strongly suppressed and the number of TRAP-positive cells in joint was also decreased by the therapeutic treated of anti-FKN mAb.

Conclusions: Anti-FKN mAb demonstrated a remarkable efficacy in the arthritis score without affecting systemic inflammatory parameters and inhibited the joint destruction with the marked reduction of osteoclasts in CIA model. These results suggest that inhibition of FKN/CX3CR1 axis by a humanized anti-FKN mAb, E6011, is an attractive therapeutic strategy for the treatment of both inflammatory synovitis and joint destruction of RA.

Disclosure of Interest: None declared

DOI: 10.1136/annrheumdis-2017-eular.3599

\section{THU0032 THE ROLE OF S100A9 IN PAIN RESPONSE DURING EXPERIMENTALLY INDUCED ACUTE SYNOVITIS}

E.J.W. Geven, E.N. Blaney-Davidson, E.L. Vitters, A.W. Sloetjes, B. Walgreen, A.B. Blom, P.L.E.M. van Lent. Experimental Rheumatology, Radboud university medical center, Nijmegen, Netherlands

Background: Synovitis-associated pain is an important aspect of arthritis pathology. Several inflammatory mediators released by the synovium have been implicated in the regulation of pain, including S100A8 and S100A9 which may regulate pain either via direct stimulation of TLR4 on the nerve endings in the synovium or via stimulation of the dorsal root ganglia (DRG), thereby enabling an increased phagocyte infiltration.

Objectives: To investigate the role of S100A9 in the pain response after induction of an acute synovitis using streptococcal cell walls (SCW) as a trigger, comparing S100A9 ${ }^{-/}$mice and their WT controls.

Methods: Acute synovitis was induced by a single i.a. injection of SCW in the knee joint of C57BI6 (WT) mice and S100 A9 ${ }^{-/-}$mice, control mice received an i.a. saline injection. Serum S100A8/A9 levels were investigated by ELISA and expression of S100A8 and S100A9 in synovium and DRG by immunohistochemistry. Joint swelling and cell influx was assessed by ${ }^{99 \mathrm{~m}} \mathrm{Tc}$ accumulaiton and histology, respectively. Pain response were investigated Incapacitance Tester (weight bearing), Catwalk (gait analysis) and von Frey's filaments (mechanical allodynia). Gene expression of inflammatory mediators and neuron activation markers in DRG were determined by q-PCR.

Results: A single i.a. injection of SCW resulted in increased synovial expression of S100A8 and S100A9 and subsequent increased serum S100A8/A9 levels (2.6-fold, $P<0.001) 1$ day p.i., which returned to basal levels at 7 days p.i. The increased expression of S100A9 did not contribute to the development of inflammation since joint swelling and cell influx were similar in WT and S100A9 ${ }^{-1-}$ mice 1 day p.i. Using the Incapacitance Tester, WT mice showed a marked and significant decrease in percentage of weight bearing on the SCW injected hindpaw $(28 \%)$ compared to saline injection $(47 \%, P<0.001) 1$ day p.i., whereas $\mathrm{S} 100 \mathrm{~A}^{-/-}$mice did not. In addition, gait analysis showed that the stand-phase of the unaffected paws were significantly increased in WT mice 1 day p.i. which will reduce the load on the inflamed paw, while in $\mathrm{S} 100 \mathrm{Ag}^{-/-}$mice these parameters were not altered. No difference in mechanical allodynia was observed, both mouse strains showed a similar reduction of paw withdrawal threshold $(4.2$ and 4.5 fold decrease respectively). Analysis of DRG showed no increased phagocyte infiltration after SCW injection as determined by S100A8 and S100A9 immunohistochemistry and no change in gene expression of MCP-1, KC, IL-1 $\beta$ or TNF was measured. However, expression of neuron activation markers NAV1.7, ATF3 and GAP43 were significantly increased at 1 day after SCW injection in WT mice as compared to saline injected mice $(P=0.022,0.004$ and 0.030 , respectively) while SCW injection in S100 A9 $9^{-/}$mice did not show increased expression, which is in line in with the reduced pain response observed earlier in S100A9 ${ }^{-/-}$mice.

Conclusions: These findings show that S100A9, which is released from the synovium upon inflammation, is an important mediator of inflammatory pain response in the knee, and that during the acute phase of inflammation is likely regulated via direct activation of TLR4 on nerve endings in the synovium and not via increased infiltration of phagocytes in the DRG.

Disclosure of Interest: None declared

DOI: 10.1136/annrheumdis-2017-eular.5800

\section{THU0033 LOCAL INJECTION OF ADIPOSE-DERIVED MESENCHYMAL STROMAL CELLS IN EXPERIMENTAL INFLAMMATORY OA RESULTS IN INTERLEUKIN-1 $\beta$-MEDIATED ATTRACTION OF PMNS AND REDUCED S100A8/A9 RELEASE}

S. van Dalen, A. Sloetjes, M. Helsen, B. Walgreen, E. Geven, P. van Lent. Experimental Reumatology, Radboud university medical center, Nijmegen, Netherlands

Background: Recent studies have shown that mild synovitis in early phases of osteoarthritis $(\mathrm{OA})$ is conducive to development of joint damage. OA synovitis is characterized by elevated levels of pro-inflammatory factors like S100A8, S100A9, interleukin-6 (IL-6), and interleukin-1 beta (IL-1 $\beta$ ). S100A8/A9 was found to be crucial in mediating joint destruction in inflammatory experimental $O A$. Previously we found that adipose-derived mesenchymal stromal cells (ASCs) exhibit immunosuppressive characteristics and reduce joint pathology after local application into mouse knee joints with experimental inflammatory OA. This protective effect is only perceived after intra-articular injection in early but not late stage $O A$, suggesting that the effect may be mediated by an inflammatory milieu. Objectives: To examine the working mechanism of ASCs after early injection in experimental OA.

Methods: Experimental OA was induced by injection of collagenase into murine knee joints (CiOA). Total knee joints were stained with haematoxylin/eosin and the PMN-specific antibody NIMP-R14. ASCs were isolated from murine adipose tissue and stimulated for $24 \mathrm{~h}$ with IL-1 $\beta$ or S100A8/A9. Gene expression in stimulated cells was analyzed using qPCR. Protein levels of chemokines and cytokines were measured in culture supernatant using Luminex. Migration of MACS isolated bone marrow (BM-) PMNs towards ASC-conditioned medium (CM) was examined using Transwell inserts. ASCs were co-cultured with BM-PMNs and analyzed using histology and Luminex.

Results: ASC injection into day 7 CiOA knee joints (when synovitis and IL-1 $\beta$ and S100A8/A9 levels are highest) caused a strong attraction of mainly PMN-like cells and their clustering around ASCs in the synovium shortly after injection (6h), which was confirmed by immunohistochemistry. IL-1 $\beta$ stimulation of ASCs in vitro strongly increased gene expression of PMN-attracting chemokines KC, CXCL5, and CXCL7 as well as protein levels of KC, whereas S100A8/A9 did not. The migration of BM-PMNs through Transwell inserts towards CM of IL-1 $\beta$-stimulated ASCs was significantly increased (from $5 \%$ to $10 \%$ ) when compared to CM of non-stimulated ASCs. Next, ASCs were co-cultured with BM-PMNs in the presence or absence of IL-1 $\beta$. After $6 \mathrm{~h}$, a clear clustering of neutrophils around ASCs was observed, with a significant increase in the number of ASCs clustering with PMNs, as well as a significantly elevated number of clustering PMNs per ASC after IL-1 $\beta$ stimulation. Interestingly, association of PMNs with ASCs lead to a significantly lowered release of KC protein by ASCs $(69 \%$ and $76 \%$ lower after $24 \mathrm{~h}$ and $48 \mathrm{~h}$ respectively), as well as a significantly reduced release of S100A8/A9 protein by the PMNs. This coincided with lowering of S100A8/A9 levels in washouts of inflamed synovium $6 \mathrm{~h}$ and $48 \mathrm{~h}$ after injection of ASCs in day 7 CiOA knee joints.

Conclusions: Local application of ASCs in inflamed CiOA knee joints results in attraction and clustering of PMNs with ASCs in the synovium. This presumably runs via IL-1 $\beta$-mediated up-regulation of chemokine release by ASCs, and ultimately results in significantly lowered S100A8/A9 levels.

Acknowledgements: This research was supported by the Dutch Arthritis Association.

Disclosure of Interest: None declared

DOI: 10.1136/annrheumdis-2017-eular.5841 